\title{
Classification of Video Shots Based on Human Affect
}

\author{
（人間の感情に基づくビデオショットの分類）
}

Kok-Meng $\mathrm{Ong}^{\dagger}$ and Wataru Kameyama (member) ${ }^{\dagger}$

\begin{abstract}
This study addresses the challenge of analyzing affective video content. The affective content of a given video is defined as the intensity and the type of emotion that arise in a viewer while watching that video. In this study, human emotion was monitored by capturing viewers' pupil sizes and gazing points while they were watching the video. On the basis of the measurement values, four features were extracted (namely cumulative pupil response (CPR), frequency component (FC), modified bivariate contour ellipse area (mBVCEA) and Gini coefficient). Using principal component analysis, we have found that two key features, namely the CPR and FC, contribute to the majority of variance in the data. By utilizing the key features, the affective content was identified and could be used in classifying the video shots into their respective scenes. An average classification accuracy of $71.89 \%$ was achieved for three basic emotions, with the individual maximum classification accuracy at $89.06 \%$. The development in this study serves as the first step in automating personalized video content analysis on the basis of human emotion.
\end{abstract}

Key words: Emotion, Video Content Classification, Pupil, Gaze

\section{Introduction}

Video content analysis has attracted efforts of researchers from various disciplines. The demand is mainly due to the fast growing volume of digital video in both the professional and consumer environment ${ }^{11}$. Video content can be generally perceived in two basic levels, namely cognitive level and affective level ${ }^{2}$. Cognitive level contents are the semantics that describe the "facts," e.g., the structure of the story, the composition of a scene and the objects and people captured by camera and etc. Affective level contents, on the other hand, are defined as the amount and type of affect (feeling or emotion) that are contained in video, and are expected to arise in users while watching that video ${ }^{23)}$. In this paper, our major focus is on the affective content of video.

Major effort in affective video content analysis in recent years is based on 'Signal-Based Approach'. Researchers have tried to extract the affect of digital video based on low level audio and video signals. For example, the length of video shot, the color histogram, the frequency of the background audio and etc ${ }^{2) 4)-6)}$. However, the signal based approach suffers from a major

Received November 28, 2008; Revised March 2, 2009; Accepted March 25,2009

$\dagger$ Graduate School of Global Information and Telecommunication Studies, Waseda University

(1011 Nishi-Tomida, Honjo-shi, Saitama, 367-0035 Japan) drawback, i.e. human emotion response is actually originated from human being. The methodology of affective content analysis has to differ from conventional 'SignalBased Approach' because it needs to take into account the feeling of human, who is the actual viewer of the video content. In addition, although a generalized approach could be applied for majority of people, because some difference exists from one individual to another, a personalized approach has to be applied for some specific individual. Therefore, we have adopted another approach, that is a viewer-oriented approach, which attempts to determine the affective content of video based on viewer's point of view.

In this paper, the problem of extracting the affective content is treated as the problem of classifying the shots into their belonging scenes with distinct emotion label. The latter implies that we determine whether a shot belongs to a specific group of emotion. Since a scene is composed of many shots, the classification of the shots requires high-level concepts connecting the scene's affective content and subjects' emotion. In order to achieve this, we propose an approach to extract the human affective content by using measurement of the pupil size and gazing point. By classifying the video shots by them into their respective scene, we demonstrate that this approach can be a first step in realizing the automation of personalized video content analysis based on human emotion. 
These types of affective classification are beneficial to video indexing and retrieval application. A tool capable of interpreting video in such a way would be a great enhancement for the recommendation processes in future video-on-demand systems or personal video recorders. There are two scenarios where our proposal could be applied. First is for a general application, where a database of pupil size and gazing point for an appropriate sample size of people is to be collected for video archives in controlled environment. Then, the video archives are to be automatically indexed based on our proposal. The indexed video archives could then be retrieved by public users using the affective keywords. In fact, viewers often select a video to watch because it is exciting, or make us feel good or happy. In addition, parental control can be exercised to apply restriction to the horror video that is deemed unsuitable for their young ones. And of course, neutral videos would be skipped when viewers are on tight schedule.

As the second scenario, our proposal can be applied for ambient intelligence, where a home video system could recommend an appropriate video according to the mood and feeling of the viewer. To achieve this, practically the viewer's personalized pupil size and gazing point data has to be collected. With the advent of miniaturization technology, head mounted type eye tracker has gone as light as 76 grams $^{7)}$. Furthermore, extensive research is being carried out in eye tracking system that do not require the viewers to put on any device. For example, there is a research using only passive eye images ${ }^{8)}$ to determine the gaze. In this case, no equipment has to be attached to the viewer while their data is being collected. According to Picard ${ }^{9}$, the affective wearable in future will be thoroughly non-invasive and measure the wearers' signal without faintest disturb to their daily activities. Therefore, we foresee in the near future, the viewer's pupil size and gazing point data can be collected without any disturbance to their daily life.

The rest of the paper is organized as follows. The next section overviews related works and clarifies some fundamental issues. Section 3 details our proposed method. Section 4 explains our experimental setting. Section 5 presents our results and consideration, and finally the paper is concluded in Section 6.

\section{Related Works and Fundamental Is- sues}

\subsection{User-centric Affective Video Content Analysis}

Toyosawa et al. ${ }^{11)}$ use heart rate based arousal level as weights to determine the video shots that deem impressive and consuming. A StartleCam based on skin conductivity signal is introduced by Haeley et al. ${ }^{12)}$. Aizawa et al. ${ }^{13) 14)}$ propose automatic summarization on the video data captured using their Life Log system. Segments of videos are evaluated by brain waves. As pointed out by Toyosawa et al. ${ }^{11)}$, these works have a major problem in common: sensors are required to be attached to the viewer. In addition, physiological signals like Galvanic Skin Response (GSR) and Electromyography (EMG) are susceptible to noise when the viewer performs physical movement during the measurement.

The natural solution to these problems is the eye. The eye is the organ that must be used by the viewers while watching video content. In this research, two parameters from the eye, namely the pupil size and gazing point are used. Pupil dilation is a good indicator of novelty, interest, positive evaluation, and also cognitive load, whereas decrease in pupil size indicates fatigue and possibly negative stimuli ${ }^{17)}$. Recently, Partala et al. ${ }^{18)}$ reported that pupil size is significantly larger for both positive and negative stimuli, as opposed to neutral ones.

On the other hand, information obtained by tracking the gaze of users is also of increasing interest because it represents the interests that users have for specific objects. It is reported that viewers have tendency to look at the similar center of interest ${ }^{19)}$. Fixation or visual fixation is the maintaining of visual gaze on a location, and the brain assigns semantics only during the eye fixation ${ }^{20)}$. Thierry et al. $^{20)}$ present fixation identification technique that is based on clustering of eye positions, using projections clustering and projection aggregation applied to static pictures. Grounded upon these findings, we extract features from pupil size and gazing point, because we believe they provide better fidelity to the subjects' instantaneous affective feedback, as opposed to conventional post-view evaluation such as questionnaire.

\subsection{Influence of Brightness on Pupil Size}

The diameter of the pupil is sensitive to the amount of light falling on the eye. In order to obtain the inter- 


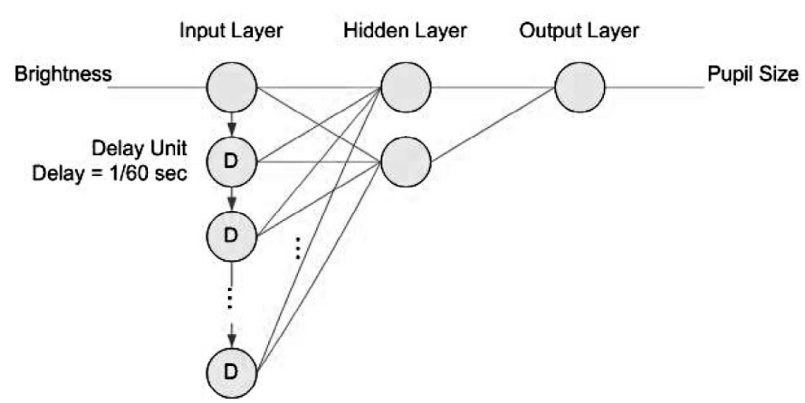

Fig. 1 Neural Network Model for Brightness Compensation.

est level from the pupil size, it is crucial to correct the measured pupil size from the pupillary change that is caused by variation in brightness.

Due to the nature of dynamic brightness level in video, we have to compensate the pupillary change that is caused by brightness change in the video. According to Shimizu et al. ${ }^{21)}$, this pupillary change can be modeled. They have compared two models, namely the linear and the neural network models, and found that the neural network model outperforms the linear model in modeling pupillary response to brightness change. They have concluded that best performing network is the multilayer (3 layers) perceptron neural network with the following structure:

- Input Layer: Brightness of current and previous frames. The best result is obtained between 25 to 40 input nodes, which is corresponding to between 0.83 to 1.33 seconds of pass brightness data. They have reasoned that this is due to the latency in pupillary response and the response peaks at around 1.0 second $^{21)}$

- Hidden Layer: Best result is achieved with 2 hidden nodes

- Output Layer: 1 output node, which represents the pupil size.

In addition, among 8 input sequences that were used, they have reported that the best trace of pupil response happens when the input is square wave with period of 2 seconds.

Therefore, to emulate the neural network that is proposed in that paper, a multilayer (3 layers) perceptron neural network with the following structure is used in this paper: 60 input nodes are used at the input layer, which are the brightness level for the past 1 second of data (In our case, the sampling frequency of pupil is $60 \mathrm{~Hz}$ ). There are 2 nodes in the hidden layer and the output node represents the pupil size. The neural network is depicted in Fig. 1

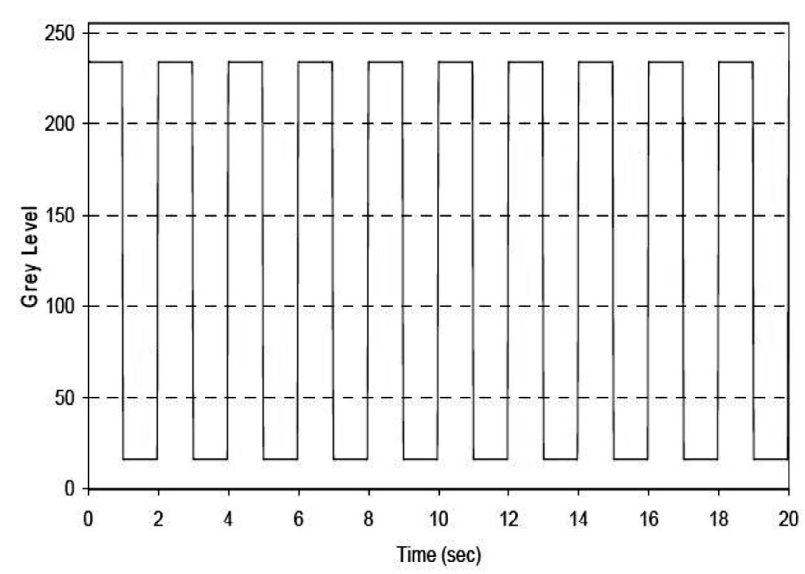

Fig. 2 Brightness Profile of Calibration Sequence for Neural Network Compensation.

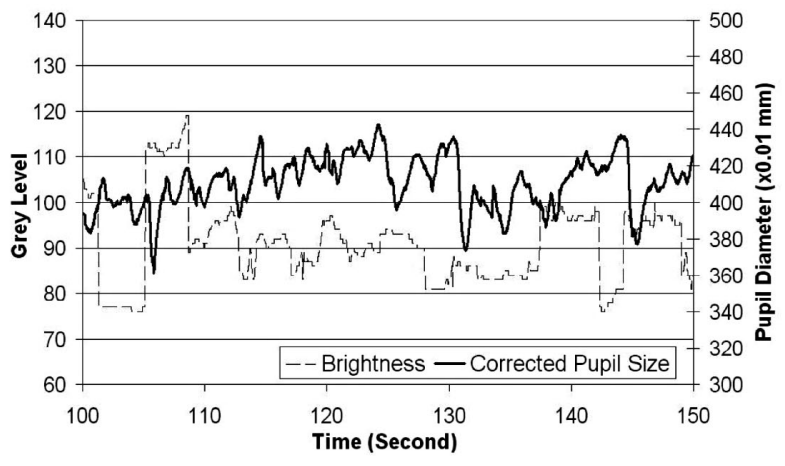

Fig. 3 Corrected Pupillary Changes due to Brightness.

The network is trained using a video calibration sequence via the back propagation algorithm. The calibration sequence is square waveform video where the brightness level is alternated continuously from high to low, with period of 2 seconds as depicted in Fig. 2

Finally, the corrected value for pupillary response due to brightness change is obtained by finding the difference between the outputs of the neural network with the baseline pupil size. The baseline pupil size is obtained by taking the average response of the whole square sequence of alternate brightness. As an example, Fig. 3 depicts the corrected value of pupillary changes due to brightness for one of the test sequence.

\section{Proposed Method}

\section{1 System Overview}

Our proposed analysis system is shown in Fig. 4. The pupil size and gazing point are measured while the subjects are watching the test video scenes. The obtained measurement values are sent for preprocessing, where eye-blink artifact is removed and neutral network compensation is applied for pupil size measurement. $\mathrm{Cu}^{-}$ mulative Pupil Response (CPR) and Frequency Com- 


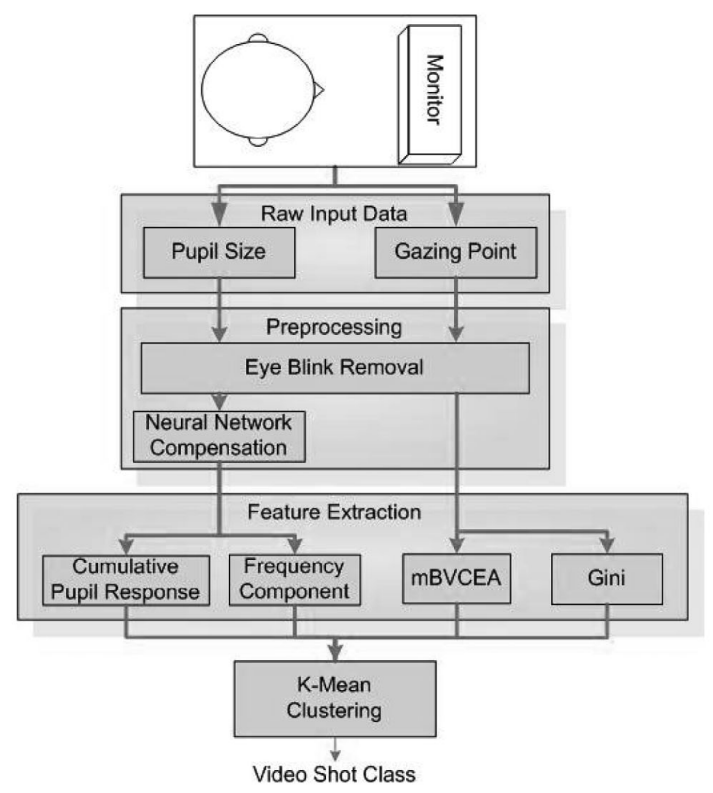

Fig. 4 System Architecture.

ponent (FC) are calculated from the preprocessed pupil size value. From the preprocessed gazing point measurement, modified Bivariate Contour Ellipse Area (mBVCEA) which represents the gaze dispersion, and Gini Coefficient (Gini) which represents the gaze concentration are calculated as features. The features are concatenated to form the input for each shot, which are sent to the classification using K-Means Clustering algorithm.

\subsection{Pupil Features}

Two features are extracted based on pupil size measurement:

(1) Cumulative Pupil Response, (CPR)

The instantaneous pupil measurement value can not be used as the feature because latency exists in pupillary response to stimuli. Therefore, we introduce CPR, which is given by the following formula:

$$
C P R=\sum_{t-T}^{t} \frac{P_{m}(t)-P_{b}}{P_{b}}
$$

The time window $T$ for this cumulative affect is chosen as $T=1$ second in this paper because pupil reacts to stimuli in 0.2 second, with the response peaking in 0.5 to 1.0 second $^{16)}$. $P_{m}(t)$ is the preprocessed pupil size measurement at time t. $P_{b}$ is the subject specific baseline pupil size value. It is obtained by taking the average of pupil size response of calibration sequence, as described in Section 2.2, before the data collection.

To verify the effectiveness of CPR in locating high arousal points, a preliminary experiment was carried out. Where 15 subjects were requested to watch an animation video. The average result of 15 subjects is

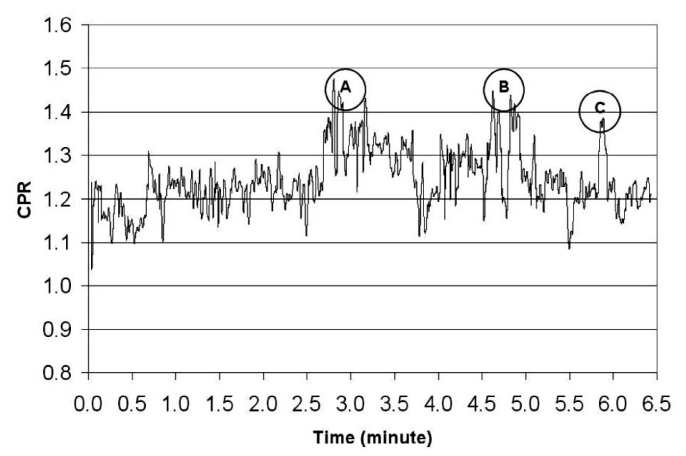

Fig. 5 Results on Cumulative Pupil Response.

shown in Fig. 5. The three region with high value of CPR are the areas where high arousals are located as expected:

- A: Sudden slapping action of a character

- B: Kissing scene

- C: Appearance of villain character

(2) Frequency Component, (FC)

The pupillary change is an indicator of the variation in the pupil size. The FC is used here as an index of the activity degree of the pupil size. The pupil size data is truncated into 2 second window to obtain the Fourier Transform $X$. The pupilogram is generally low pass filtered (below $4 \mathrm{~Hz}$ ) because it is a biological signal ${ }^{15}$. In addition, signal from 0.05 to $0.3 \mathrm{~Hz}$ are considered pupillary noise ${ }^{15)}$. Therefore, only the frequency component from $0.3 \mathrm{~Hz}$ to $4 \mathrm{~Hz}$ are extracted as feature:

$$
F C=\sum_{f=0.3 H z}^{4 H z}|X|
$$

\section{3 Gazing Point Features}

In this paper, two parameters are used to quantify the concentration and dispersion of fixation as follow:

(1) modified Bivariate Contour Ellipse Area, (mBVCEA)

Bivariate Contour Ellipse Area, BVCEA was initially introduced in by Steinman et al. ${ }^{24)}$ to represent the ellipse area where fixation falls. The larger the BVCEA value, the dispersion of gaze is higher and vice versa. In this paper, BVCEA is modified to show the dispersion of gazing point as follow:

$$
m B V C E A=2 k \pi S_{X} S_{Y}\left(1-\rho^{2}\right)^{\frac{1}{2}}
$$

Where $S_{X}$ and $S_{Y}$ are the standard deviations of $X$ and $Y$ coordinates of gazing points, $\rho$ is the productmoment correlation of the $X$ and $Y$ gazing point coordinates, and $k$ is constant that establishes the confidence limit for the ellipse. $k=1$ is used so that the probability of the gazing points falling within the ellipse achieves 


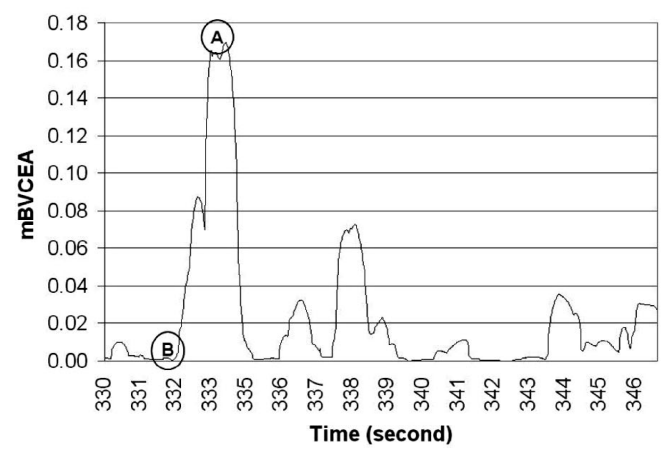

Fig. 6 mBVCEA as Indicator of Gaze Dispersion.

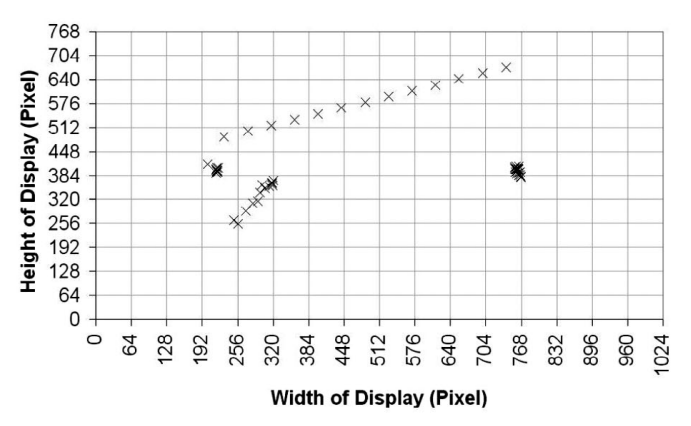

Fig. 7 High mBVCEA at A: Actual gazing points for 1 second of data - high dispersion.

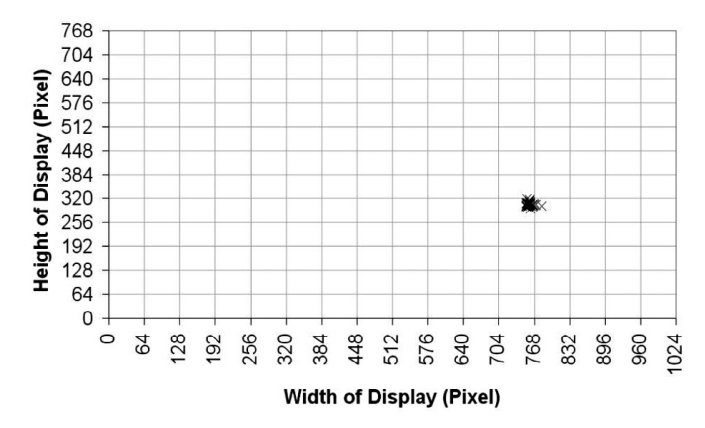

Fig. 8 Low mBVCEA at B: Actual gazing points for 1 second of data - low dispersion.

$63.2 \%$. To be consistent with $T=1$ second in CPR, the mBVCEA is computed for time window of $T=1$ second, which is equal to 60 gazing points.

A part of mBVCEA value for a test subject is demonstrated in Fig. 6. The actual gazing points are depicted in Fig. 7 and Fig. 8, showing 1 second of data, which is equivalent to 60 gazing points. The test video sequences are shown to the viewer using a monitor with resolution $1024 \times 768$ pixels. The $\mathrm{X}$-axis represents the width of the display in pixel, and the $\mathrm{Y}$-axis represents the height of the display in pixel. It is clear that when mBVCEA value is high (Fig. 7), the dispersion of gaze is high. In contrary, when then dispersion of gaze is low (Fig. 8), the mBVCEA value is low.

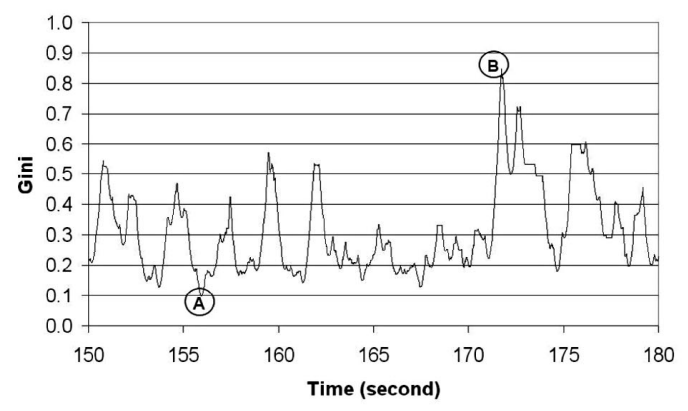

Fig. 9 Gini as Indicator of Gaze Concentration.

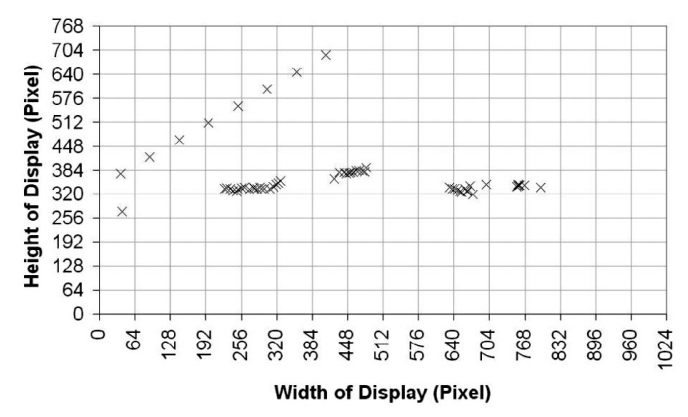

Fig. 10 Low Gini at A: Actual gazing points for 1 second of data - low concentration.

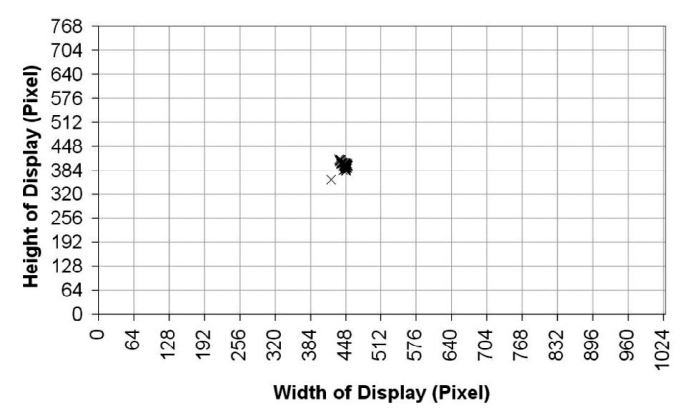

Fig. 11 High Gini at B: Actual gazing points for 1 second of data - high concentration.

\section{(2) Gini Coefficient, (Gini)}

The Gini coefficient is a measure of statistical dispersion most prominently used as a measure of inequality of income distribution in the field of economy ${ }^{20)}$. It is defined as a ratio with values between 0 and 1: A low Gini coefficient indicates more equal distribution, while a high Gini coefficient indicates more unequal distribution. In order to use this theory in evaluating gaze concentration, the computation of the Gini coefficient which is introduced by Thierry et al. ${ }^{20)}$ is adopted as follow:

$$
\text { Gini }=\sum_{i=1}^{p} \sum_{j=1}^{q} \frac{n_{i j}^{2}}{n^{2}}
$$

In regards to our case, to be consistent with $T=1$ second in CPR, the computation is adapted for time 
window of $T=1$ second. Where a frame is partitioned in $p \times q$ cells. In this experiment, $p=q=16$ pixels is used. $n_{i j}$ is the number of gazing points in $\operatorname{cell}(\mathrm{i}, \mathrm{j})$; $n=60$ is the total number of points in $T=1$ second.

To illustrate the interpretation of Gini, a part of Gini value for a test subject is demonstrated in Fig. 9. The actual gazing points are depicted in Fig. 10 and Fig. 11 for 1 second of data. This time, when Gini value is high (Fig. 11), the concentration of gaze is high. In contrary, when then concentration of gaze is low (Fig. 10), the Gini value is low.

\section{4 K-Mean Clustering}

To evaluate the efficiency in classification of the video shots, K-Mean Clustering algorithm is employed as the classifier. The output cluster label is determined by the Majority Vote ${ }^{25)}$. Each cluster is tagged with the class label that most commonly occurs among its members, and any instances in the cluster that have different class labels are considered errors.

\section{Experimental Setting}

\section{1 Test Video Scenes}

Selection of test video scenes has been a challenging task as there is little agreement on the basic emotion even among emotion theorists ${ }^{26)}$. Conventionally, researchers approach emotion in discrete domain. For example, the basic emotions has been proposed by Ek$\operatorname{man}^{26)}$. More recently, researchers model human emotion using dimensional approach. Emotion is portrayed in two dimensional arousal (level of excitement) and valence (level of pleasantness), and pictures with different affective states are charted ${ }^{10)}$. A series of experiments has been conducted by Lang et al. ${ }^{10)}$ using database of photographs as emotional stimulus. Over hundreds of subjects participated in their study and they manage to associate the stimuli pictures to various locations in the Valence-Arousal dimension. For example:

- Pictures like basket, which are associated with neutral emotion is located at low arousal with neutral valence

- Pictures like baby face, which are associated with happy emotion is located at moderate arousal with positive valence

- Pictures like snake and mutilated face, which are associated with fearful emotion is located at high arousal but negative valence

Based on this foundation, to emulate the dimensional approach yet retain the generosity in discrete emotion, three test video scenes which are located at different
Table 1 Test Scenes.

\begin{tabular}{c|c|l|l}
\hline Scene & Arousal & Valence & Description \\
\hline \multirow{3}{*}{1} & Low & Neutral & $\begin{array}{l}\text { Neutral Scene } \\
\text { Movie "The Exorcism of Emily } \\
\text { Rose" } \\
\text { From 16:37 to 18:59 } \\
31 \text { video shots, 142 seconds }\end{array}$ \\
\hline \multirow{3}{*}{3} & Moderate & Positive & $\begin{array}{l}\text { Happy Scene } \\
\text { Movie "Ratatouille" } \\
\text { From 63:01 to } 65: 41 \\
52 \text { video shots, 160 seconds }\end{array}$ \\
\hline & Low & Neutral & $\begin{array}{l}\text { Horror Scene } \\
\text { Movie "The Exorcism of Emily } \\
\text { Rose" } \\
\text { From } 31: 25 \text { to } 34: 25 \\
50 \text { video shots, 180 seconds }\end{array}$ \\
\hline
\end{tabular}

locations of the arousal-valence dimension are selected. The first scene is conversation scene in a courtroom, the second scene is a happy ending scene of an animation, and the third scene is horror scene where the character is pursued by demon. The detail description is shown in Table 1.

\subsection{Subject}

6 volunteers (3 male, 3 female), all students from Waseda University, participated in the experiment. The age of the subjects ranged from 25 to 33 years (mean 28.67; S.D. 3.26). The impairment of pupillary response has been reported for patients with psychiatric disorders ${ }^{27)}$. Therefore, all the subjects were confirmed healthy through self-reporting questionnaire. The subjects were all medication-free and were either non-alcohol consumer or occasional social alcohol consumers. The subjects were required to self-rate their level of understanding in both English and Japanese. The test video scenes were dubbed in either English or Japanese and were presented to the subjects according to their self-rated understanding and preference of the languages.

\section{3 Apparatus}

As shown in Fig. 12, a Display PC which hosts a standard 17 inches $(42.5 \mathrm{~cm})$ color monitor was used to administer the video viewing task and store the measured pupil size and gazing point. A second Control $\mathrm{PC}$ was used to control the Measurement Machine and relay the measurements obtained to the Display PC. A Subject was seated in an armless chair, facing the monitor with his/her head approximately maintaining a distance of 70 centimeter between the subject and the monitor. Pupil size and gazing point were measured from the participant's master eye using VIS-EYE Measurement Machine provided by Visual Interactive Sensitivity Research Institute Co. Ltd., Japan ${ }^{28)}$. The pupil size and gazing point were digitized at $60 \mathrm{~Hz}$ sampling rate and saved for offline processing. For consistency 


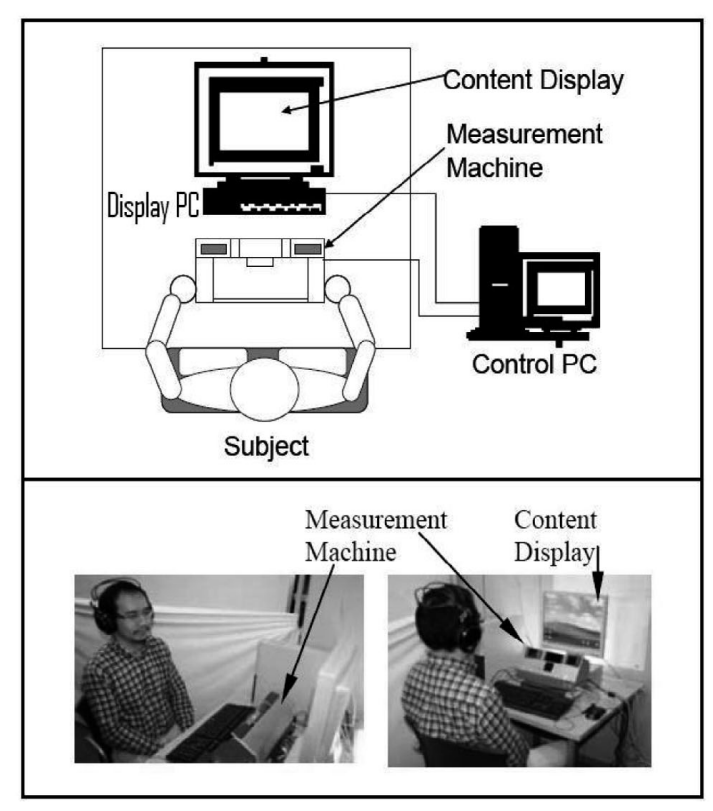

Fig. 12 Experimental Setup.

with the literature, the used pupil size is the diameter. The resolution of the pupil size was $0.01 \mathrm{~mm}$.

\section{4 Procedure}

The subjects were first briefed about the experiment. They were told that selected video scenes would be shown to them. They were also instructed to watch the video scenes at ease in order to simulate the exact situation when they watch video at leisure. The subjects were asked to take a break if they feel fatigue or tired during the experiment. The calibration was performed by instructing the subject to fixate nine points in the screen area. After that, the subjects were shown a square wave sequence with high and low brightness for the purpose of correcting the change of pupil size due to the change in video content brightness as mentioned in Section 2.2. Then, the subjects were shown the video scene which was dubbed to the preferred language. The ambient brightness was maintained at a constant level throughout the experiment. Finally, the subjects were freed from the equipment.

\section{Results and Consideration}

\section{1 Results}

During the first run, all the features (CPR, FC, mBVCEA and Gini) in Section 3 are used to form the input for K-mean Clustering. The shot is considered correctly classified if the shot label is similar to the Cluster label determined by Majority Vote ${ }^{25)}$ as mentioned in Section 3.4. Classification accuracy is obtained by taking the ratio of the number of correctly classified shots to the total number of shots. The classification accuracy of
Table 2 Classification Results Using all Features.

\begin{tabular}{c|c}
\hline Subject & Accuracy \\
\hline 1 & $37.07 \%$ \\
\hline 2 & $53.57 \%$ \\
\hline 3 & $40.32 \%$ \\
\hline 4 & $47.58 \%$ \\
\hline 5 & $39.84 \%$ \\
\hline 6 & $43.59 \%$ \\
\hline Average & $43.66 \%$ \\
\hline Random & $30.75 \%$ \\
\hline
\end{tabular}

Table 3 Variance Contribution of two Principal Component and Features with Highest Weight Associated with First PC.

\begin{tabular}{|c|c|c|c|c|}
\hline \multirow{2}{*}{ Subject } & \multicolumn{3}{|c|}{ Variance Contribution of 2 PCs } & \multirow{2}{*}{ features } \\
\hline & First PC & Second PC & Both & \\
\hline 1 & $69.58 \%$ & $23.56 \%$ & $93.14 \%$ & $\mathrm{CPR}, \mathrm{FC}$ \\
\hline 2 & $66.03 \%$ & $26.39 \%$ & $92.42 \%$ & $\mathrm{CPR}, \mathrm{FC}$ \\
\hline 3 & $66.52 \%$ & $25.48 \%$ & $92.00 \%$ & $\mathrm{CPR}, \mathrm{FC}$ \\
\hline 4 & $66.68 \%$ & $25.47 \%$ & $92.15 \%$ & $\mathrm{CPR}, \mathrm{FC}$ \\
\hline 5 & $64.17 \%$ & $24.78 \%$ & $88.95 \%$ & $\mathrm{CPR}, \mathrm{FC}$ \\
\hline 6 & $67.26 \%$ & $25.11 \%$ & $92.37 \%$ & CPR, FC \\
\hline
\end{tabular}

the clustering is tabulated in Table 2. For the purpose of comparison, accuracy of randomly generated class is shown in the next row. It is easily noticed that the classification results is slightly better than random selection.

As mentioned earlier, researchers has been representing emotion in 2-dimensional space of valence and arousal. Therefore we would like to search for the optimum two feature-pairs that can represent the arousalvalence in emotion dimension. To do this, Principal Component Analysis is employed in order to understand the data. First, the input features for all the video shots, data $X$ is arrange into matrix:

$$
X=\left[x_{C P R} x_{F C} x_{m B V C E A} x_{G i n i}\right]
$$

To find the Principal Component matrix, the covariance matrix, $A$ of $X$ is calculated. The matrix of eigen vector $E$ and eigen value $D$ is obtain using identity:

$$
A E=D E
$$

The principal component is then obtained from the column of eigen vector $E$. The variance of the corresponding PC is associated with the eigen value in $D$. The variance contribution of the two PCs are tabulated in Table 3. Based on the criteria suggested by Jolliffe $^{29)}$, the two features associated with the highest weight in the first PC is tabulated in the table too. Overall, it can be concluded that with the inclusion of CPR and FC as features, most of the variance of the data could be captured.

Based on the findings of PCA analysis, the clustering is rerun with CPR and FC as input features. The 
Table 4 Classification Results Using CPR and FC as Input Features.

\begin{tabular}{c|c}
\hline Subject & Accuracy \\
\hline 1 & $75.86 \%$ \\
\hline 2 & $66.96 \%$ \\
\hline 3 & $80.65 \%$ \\
\hline 4 & $70.97 \%$ \\
\hline 5 & $89.06 \%$ \\
\hline 6 & $47.86 \%$ \\
\hline Average & $71.89 \%$ \\
\hline
\end{tabular}

results is shown in Table 4. It is observed that the classification accuracy is significantly increased. The highest accuracy is achieved for Subject 5 at $89.06 \%$. The distribution and centroid of the k-mean cluster is shown in Fig. 13. The average classification accuracy of $71.89 \%$ is obtained. It is significantly greater than random selection.

\subsection{Consideration}

From the results, it is clear that with the inclusion of $\mathrm{CPR}$ and $\mathrm{FC}$, a promising classification results could be obtained. The CPR, which represents the pupil size response, is anticipated to be able to be utilized as indicator of arousal level of video, as it has been reported that dilation of pupil size is correlated to the rise in arousal.

However, the study of FC has been scarce in this area. It has been reported that the power spectrum density measurements from the estimated pupillography during oral calculation task increases with task difficulty in certain frequency band ${ }^{30}$. However, no further report is mentioned regarding the frequency content with human emotion. Ohtsuka et al. ${ }^{31)}$ have studied the respiratory fluctuation of human pupil and reported that pupil fluctuations is observed during respiration and the amplitude of these pupillary changes is closely proportional to the tidal volume. Extensive review on emotion-respiration relationship has been reported and it is concluded that emotions are linked to respiration ${ }^{32)}$. Therefore, we believe that the indirect link here (pupil fluctuations-respiration-emotion) plays a role for FC to contribute to the classification accuracy.

From the results, it is found out that for most of the subjects, the inclusion of mBVCEA and Gini do not contribute to higher classification accuracy. We believe that understanding of the underlying content is crucial to make mBVCEA and Gini useful in classification. For example, in a conversation scene between two characters, a viewer tends to alternate his/her gaze between the characters. This will cause high dispersion in the features, but the user is still paying high attention or is interested. Therefore, it is our next task to incorporate the relationship between the 'Signal-Based Approach' with the gazing point measurement to improve the results.

One interesting observation we would like to point out is the difference in result for every individual. This will not cause major problem in the general application when large database is collected as the video archives will be indexed based on the affect from majority. However, for the personalized application, based on our proposal, a problem of deficiency in the classification will occur. For example, the classification using CPR and FC for Subject 6 is only $47.86 \%$. In this situation, only less than half the shots will be correctly classified and the video recommendation system might not be able to make suggestion appropriately. To solve this problem, the other feature-pairs are tested and we found that by using FC and Gini, the classification results for Subject 6 has increased to $70.09 \%$. Therefore, although CPR and FC are found important for most of the viewers, because the existence of individual difference in viewerbased approach, a static classifier might not be suitable for some individual. In cases like this, a dynamic system which will customize the best features set should be employed, like the case of Subject 6 .

Furthermore, although we would like to find featurepairs that could represent the arousal-valence pair, based on the results, it seems that the we could only obtain arousal information at the moment because according to the distribution of centroids for Scene 1(Neutral), Scene 2(Happy) and Scene 3(Horror) in Fig. 13, their position changes from low, medium to high for both the axes, corresponding to the arousal level. However, we could not relate it well to the valence level which changes from neutral, positive to negative. Therefore, it is our future task to find out parameters that could be used to model the valence in video content.

\section{Conclusion}

To contribute to better affective understanding in video, this paper proposes to classify the video shots based on pupil size and gazing point during video watching. Features are extracted and classification is performed using K-Mean clustering algorithm. Two important features have been identified, they are CPR and FC. The accuracy of classification based on the two features is promising. We have achieved maximum accuracy of $89.06 \%$ for an individual subject and average accuracy of $71.89 \%$. It is our next task to incorporate the 


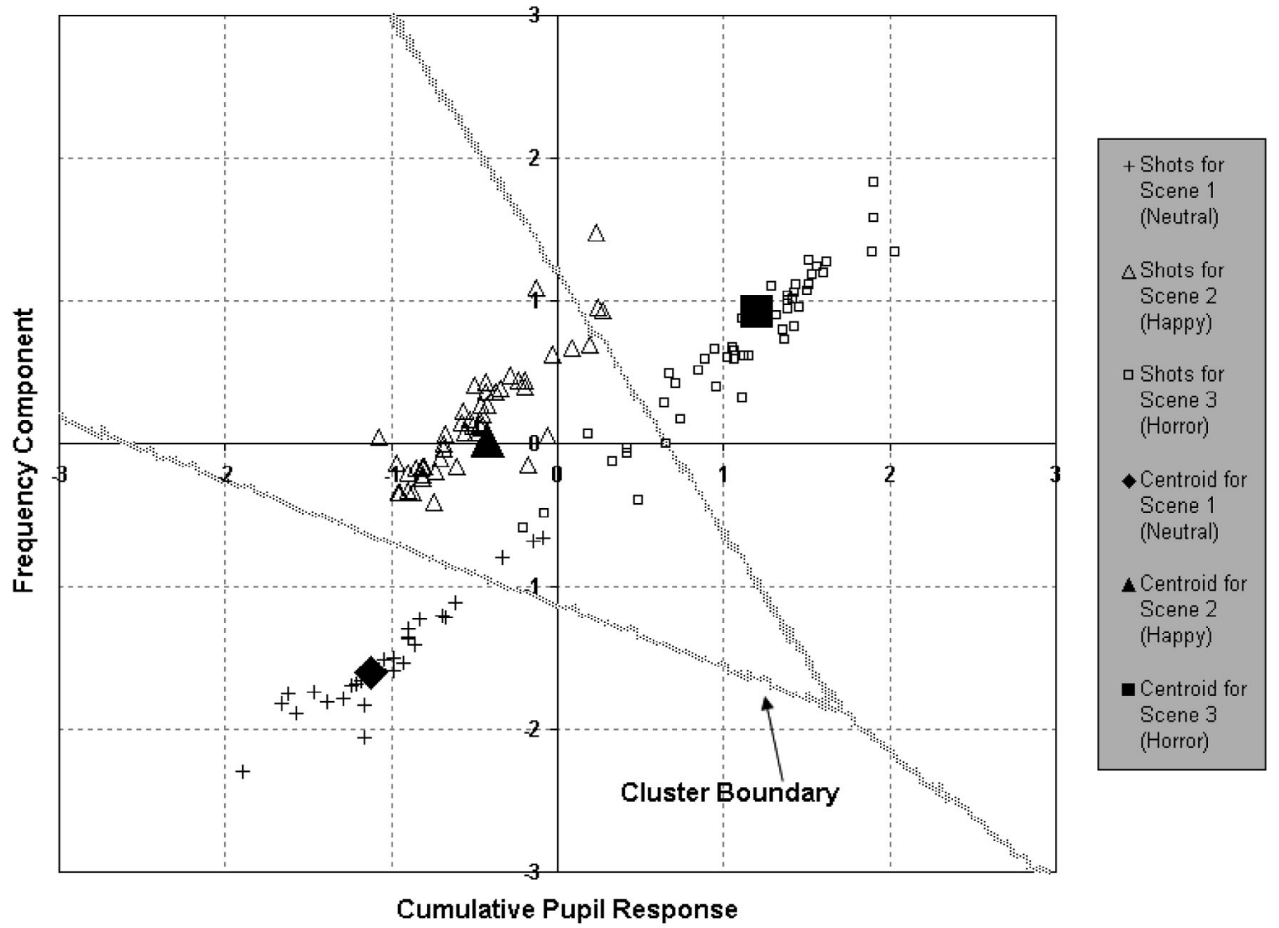

Fig. 13 Distribution of Centroid for Subject with Highest Classification Accuracy.

'Signal-Based Approach' with the gazing point features to improve its applicability in affective video content analysis. Further investigation is needed to find features that could be related well to emotion in valence dimension.

This research has been conducted jointly with VIS Research Institute.

\section{〔References}

1) Ba Tu Truong and S.Venkatesh, Video Abstraction: A Systematic Review and Classification, ACM Transactions on Multimedia Computing, Communications and Applications, 3, 1, Article 3 (Feb. 2007)

2) A. Hanjalic, Li-Qun $\mathrm{Xu}$, Affective Video Content Representation and Modelling, IEEE Transactions on Multimedia, 7, 1 (Feb. 2005)

3) A. Hanjalic, Extracting Moods from Pictures and Sounds, Towards truly personalized TV, IEEE Signal Processing Magazine, pp. 90-100 (March 2006)

4) Hee Lin Wang and Loong-Fah Cheong, Affective Understanding in Film, IEEE Transactions on Circuits and Systems For Video Technology, 16, 6, pp. 689-704 (June 2006)

5) H.B.Kang, Affective content detection using HMMs ACM Multimedia, pp.259-262 (2003)

6) Z.Rasheed, Y. Sheikh, and M.Shah, On the use of computable features for film classification IEEE Transactions of Circuits and Systems For Video Technology, 15, 1, pp 52-64 (Jan. 2005)

7) Advance Science Laboratory, http://www.a-s-l.com/site/ Products/MobileEye/tabid/70/Default.aspx

8) Carlo Colombo, Dario Comanducci and Alberto Del Bimbo, Robust Tracking and Remapping of Eye Appearance with Passive Computer Vision, ACM Transactions on Multimedia Computing, Communications and Applications, 3, 4, Article 20 (Dec. 2007)

9) Rosalind W. Picard, Affective Computing, The MIT Press, 2000

10) Lang, P.J.,Bradley, M.M., Cuthbert, B.N, International Affective Picture System(IAPS): Instruction Manual and Affective Ratings, Technical Report A-6, The Center for Research in Psy- chology, University of Florida (2005)

11) Satoshi Toyosawa and Takashi Kawai, Video Digest Based on Heart Rate, Proceedings of the Seventh IASTED International Conference, Visualization, Imaging, and Image Processing, Palma de Mallorca, pp. 15-20 (2007)

12) J Healey, RW Picard, StartleCam: A Cybernetic Wearable Camera, Proceedings of Second International Symposium on Wearable Computers, Pittsburg, PA, USA, pp. 42-49 (1998)

13) Kiyoharu Aizawa, Ken-Ichiro Ishijima, Makoto Shiina, Summarizing Wearable Video, Proceedings of International Conference on Image Processing 2001, Thessaloniki, Greece. pp. 398-401

14) D Tancharoen, $\mathrm{T}$ Yamasaki, $\mathrm{K}$ Aizawa, Practical experience recording and indexing of Life Log video, Proceedings of the 2nd ACM workshop on Continuous archival and retrival of Personal Experience, 2005, Singapore, pp. 61-66

15) Minoru Nakayama, Yasutaka Shimizu, An Estimation Model of Pupil Size for Blink Artefact and ItMacro 6's Applications, ESANN 2002 proceedings, European Symposium on Artificial Neural Networks Bruges (Belgium), 24-26 April 2002, d-side Publi. ISBN 2-930307-02-1, pp.251-256

16) John L. Andreassi, Psychophysiology Human Behaviour and Physiological Response, 5th Edition, Mahwah, New Jersey, 2007

17) H. Prendinger and M. Ishizuka, Symmetric Multimodality Revisited: Unveiling Users Physiological Activity, IEEE Trans. On Industrial Electronics, 54, 2, pp. 692-698 (April 2007)

18) T. Partala, V. Surakka, Pupil size variation as an indication of affective processing, Int. J. Human-Computer Studies, 59, pp.185-198 (2003)

19) Goldstein R.B., Russel L. and Woods E.P., Where people look when watching movies: Do all viewers look at the same place, Computers in Biology and Medicine, 37, pp.957-964 (2007)

20) Thierry Urruty, Stanislas Lew, Nacim Ihadaddene, Detecting Eye Fixations by Projection Clustering, ACM Transactions on Multimedia Computing, Communications and Applications, 3, 4, Article 23 (Dec. 2007)

21) Shigeyoshi Asano, Ikki Yasuike, Minoru Nakayama, Yasutaka Shimizu, A Pupil Reaction Model with Neural Network for Brightness Change, The Journal of The Institute of Electronics, Information and Communication Engineers, J77-A, 5, pp.794801 (May 1995)

22) $\mathrm{Ba} \mathrm{Tu}$ Truong, Svetha Venkatesh and Chitra Dorai, Scene Extraction in Motion Pictures, IEEE Transactions on Circuits and Systems for Video Technologies, 13, 1, (Jan. 2003) 
23) HongJiang Zhang, Atreyi Kankanhalli, Stephen W. Smoliar, Automatic partitioning of full-motion video, Multimedia System, 1, pp.10-28 (1993)

24) Robert M. Steinman, Effect of Target Size, Luminance, and Color on Monocular Fixation, Journal of the Optical Society of America, 55, 9, pp.1158-1165

25) Kiri Wagstaff, Claire Cardie, Clustering with Instance-level Constraints, Proceedings of the Seventh International Conference on Machine Learning, 2000, pp. 1103-1110

26) Andrew Ortony and Terence J. Turner, What's Basic About Basic Emotion?, Psychological Review, 97, 3, pp. 315-331 (1990)

27) A. Minassian, E. Granholm, S. Verney, W. Perry, Pupillary dilation to simple vs. complex tasks and its relationship to thought disturbance in schizophrenia patients, Int. J. of Psychophysiology, 52, pp. 53-62 (2004)

28) http://www.visri/jp/english/index.html

29) I.T.Jolliffe, Discarding variables in a principal component analysis, II: Real Data", Appl. Statist, 22, pp. 21-31 (1973)

30) Minoru Nakayama, Yasutaka Shimizu, Frequency Analysis of task evoked pupillary response and eye-movement", Proceedings of the 2004 Symposium on Eye Tracking Research and Applications, San Antonio, Texas (2004)

31) K. Ohtsuka, K. Asakura, H. Kawasaki and M. Sawa, Respiratory Fluctuation of the Human Pupil", Journal of Experimental Brain Research, 71, 1, pp. 215-217 (Jun 1988)

32) Frans A. Boiten, Nico H. Frijda, Cornelis J.E. Wientjes, Emotions and respiratory patterns: Review and Critical Analysis", International Journal of Psychophysiology, 17, pp. 103-128 (1994)

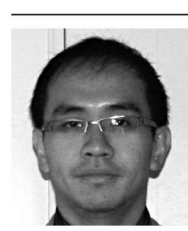

Kok-Meng Ong received a Bachelor of Electrical Engineering in 2003, and a Master of Engineering Science in 2006, both from University of Malaya, Kuala Lumpur, Malaysia. He is currently a Doctoral Course student at Waseda University, Japan.

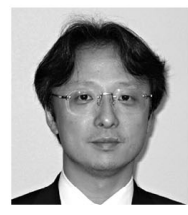

Wataru Kameyama received Bachelor. Master and Doctor of Engineering from the School of Science and Engineering, Waseda University in 1985, 1987 and 1990 respectively. He worked as a research associate at the School of Science and Engineering, Waseda University from 1989 to 1992. He joined ASCII Corp. in 1992, and was transferred to France Telecom CCETT from 1994 to 1996 for his secondment. He was an associate professor at the Graduate School of Global Information and Telecommunication Studies, Waseda University from 1999 to 2002 . He has been appointed as a professor at the Graduate School of Global Information and Telecommunication Studies, Waseda University since 2002. He is the incumbent Dean of Graduate School of Global Information and Telecommunication Studies, Waseda University. He has been involved in MPEG, MHEG, DAVIC and the TVAnytime Forum activities. He was the chairman of ISO/IEC/JTC1/SC29/WG12, and the secretariat and vice-chairman of the TV-Anytime Forum. He received the best paper award of Niwa-takayanagi in 2006 from the Institute of Image Information and Television Engineers. He is current member of IPSJ, ITE, IIEEJ, IEICE, ACM and IEEE. 